\title{
Comprensión Epistemológica e Inferencia Inductiva: Un estudio sobre la física en preescolar
}

\author{
Mario Fernando Gutiérrez Romero ${ }^{1}$
}

\author{
Colombia
}

mfgutierrez@telesat.com.co

\footnotetext{
${ }^{1}$ Este artículo proviene de la investigación Inferencia y Comprensión Epistemológica: Una perspectiva desde su microgénesis, financiada por el Centro de Investigaciones de la Universidad Antonio Nariño, Santa Fe de Bogotá. A ellos el reconocimiento por el apoyo a la investigación en Psicología.
} 


\section{Resumen}

Introducción. Esta investigación abordó la comprensión intuitiva por parte de preescolares del movimiento rectilíneo y de algunas variables que lo afectan, y el tipo de argumentos utilizados cuando se justifican estados de conocimiento relacionados. Una primera hipótesis de trabajo era que la resolución de situaciones de tipo práctico y funcional estaría basada en procesos de autorregulación. Una segunda hipótesis de trabajo era que la información de retorno que proveen las situaciones debería modificar sus niveles de comprensión epistemológica.

Método. La muestra estuvo conformada por 40 niños y niñas con una edad entre 5.0 y 5.5 años de edad seleccionados al azar, de tres instituciones de educación preescolar de carácter privado de Cali (Colombia), y asignados a dos grupos. El material experimental consistió en cuatro situaciones isomorficas basadas en una tarea de Chen y Klahr (1999).

Resultados. Los datos apoyan la primera hipótesis, el promedio de acciones aumentó significativamente entre sesiones, las estrategias emprendidas por los niños se organizaron en series más complejas y con una efectividad mayor. No se encontró un apoyo empírico a la segunda hipótesis, la mayoría de los argumentos utilizados muestran una estabilidad en la forma como describen el conocimiento de los personajes de la situación.

Discusión. En las verbalizaciones de los niños se identificó un proceso de autorregulación, pasando de mencionar las acciones emprendidas sobre el material, a controlar las acciones anticipadamente. Se discuten posiciones como las de Schauble (1996) que afirman que los preescolares son renuentes a abandonar estrategias a pesar de su inutilidad. La actividad no resultó ser efectiva para transformar los argumentos utilizados y por ende sus niveles de comprensión epistemológica.

Palabras clave: Autorregulación, Comprensión Epistemológica, Concepciones Implícitas, Física en Preescolar, Inferencia Inductiva. 


\section{Introduction}

Investigaciones sobre Desarrollo Cognitivo y Razonamiento Científico han indagado la naturaleza de las concepciones intuitivas que tienen los niños sobre ciertos dominios de conocimiento y en particular el físico (Vosniadou, 2002; Pauen, 1996). Estas concepciones implícitas han mostrado ser de gran solidez cuando se lleva a los niños a realizar juicios causales sobre hechos físicos. Dichas ideas causales sobre el mundo físico generan predicciones que siguen siendo consistentes a pesar de recibir información contradictoria, esta capacidad predictiva, y su consistencia en el tiempo, son sus rasgos distintivos que comparten con las teorías científicas en el sentido estricto (Pozo, Sanz y Limón, 1992).

Esto reviste una gran importancia en el ámbito educativo ya que estas concepciones son extraídas del mundo real y conllevan un cierto nivel de veracidad para quien las percibe, además que son generalizadas de una manera implícita, sobreponiéndose o ligándose al conocimiento que se imparte en las instituciones educativas y generando así, un híbrido que concilia de manera arbitraria y errónea ambos conocimientos.

Se crea entonces una relación funcional entre la forma como percibimos el mundo, la generalización que le damos a dicho conocimiento, y la forma como nos desempeñamos en éste. Así la transformación de dichas concepciones es un objetivo práctico de gran utilidad en el ambiente educativo, sin embargo las investigaciones en esta temática han abordado el problema desde posiciones que resultan diferentes.

Una primera ha abordado el conocimiento intuitivo que tienen los niños sobre física desde el razonamiento científico (ver Penner y Klahr, 1996; Schauble, 1996) mostrando la solidez que tienen algunas concepciones intuitivas y la relación que tienen con el desarrollo cognitivo, siendo esta una relación significativa.

Una segunda posición, basada en la necesidad de generar métodos de aprendizaje para la enseñanza de las ciencias, intenta modificar las concepciones implícitas a través de las situaciones de resolución de problemas y los contextos de indagación científica. Consideradas estrategias válidas para ser utilizadas con adolescentes, debieran ser utilizadas con el fin de acercar las concepciones de los estudiantes, a las aceptadas por el conocimiento científico socialmente válido (Chen y Klahr, 1999; Niaz, 1995; Pozo, 1993). 
Estas posiciones varían en la importancia asignada a la instrucción formal y al conocimiento anterior a la resolución de la actividad, pero coinciden en que en los salones de clase se debería impulsar el conocimiento de dominio especifico y una aproximación a la resolución de problemas y al método científico, teniendo una visión no especifica de "método científico" y considerando que la proliferación de diferentes métodos es una oportunidad para el avance de la ciencia.

Se plantea entonces, que el aprendizaje de las ciencias debe ir más allá de la memorización de hechos y formulas, menos de contenido científico y más de procesos de investigación, sintetización de nueva información y elaboración de hipótesis. Es evidente entonces que, aunque existen desde temprana edad, estas concepciones son abordadas desde una perspectiva pedagógica en la adolescencia, descuidando así a la población infantil que es en general reservada para estudios de carácter representacional.

Este artículo presenta una estrategia para transformar el conocimiento que tienen los niños menores sobre física basada en la presentación de situaciones de resolución de problemas que difieran en contenido, pero que contengan la misma estructura lógica (situaciones isomórficas). La consistencia del conocimiento infantil esta en estrecha relación con el estatus epistemológico del conocimiento intuitivo, como ya lo ha puesto en consideración Oliva (1999) y la conceptualización que se logre sobre dichas acciones.

Se descubre entonces que la capacidad para tener un control consciente sobre las estrategias utilizadas en la resolución de una situación de problemas y la forma como se relacionen los niños con su propio conocimiento, en función de los argumentos utilizados, son variables que se tornan significativas. Desde la psicología cognitiva y en particular desde el razonamiento científico existen varios conceptos que resultan útiles para analizar tales variables - la autorregulación basada en procesos metacognitivos y la comprensión epistemológica - los cuales permitirán profundizar los análisis en torno a la actividad del niño.

\section{Autorregulación y Comprensión Epistemológica}

Las actividades de autorregulación pueden considerarse como estrategias orientadas a una meta que permiten al niño seleccionar información, situándose en diferentes niveles de 
abstracción, y que suponen una capacidad para controlar su propia actividad (Lacasa, Martín, y Herranz, 1995). Se puede considerar entonces la autorregulación como una operacionalización de la metacognición pues ésta se caracteriza por reflejar, monitorear y regular procesos de primer orden (Kuhn, 2002).

Se propone entonces que la acción sobre el material produce en los niños diferentes niveles de abstracción al extraer información que va más allá de la información dada, esta es una capacidad que surge de un proceso cognitivo estudiado ampliamente en los últimos años: la inferencia. Particularmente la inferencia inductiva y funcional se propuso en esta investigación en tanto está ligada a la manipulación directa de los mecanismos, y la información significativa para la resolución de la misma surge en la medida en que se comprende la función de los elementos (Ordóñez, 2000).

Lo inductivo surge de la identificación de una regularidad en un patrón de datos o conjunto de evidencia dándole forma de hipótesis o regla explicativa a la variación de tales datos (Florian, 1994). El desempeño de niños ante situaciones que involucren inferencia inductiva ha sido caracterizado desde varias perspectivas y se ha dicho que probablemente sea el único proceso que genere conocimiento verdaderamente nuevo (Jhonson-Laird, 1994).

Los estudios realizados sobre inferencia inductiva abarcan la predicción de vectores de movimiento generado por al menos dos fuerzas (Pauen, 1996), las explicaciones causales de movimiento animado e inanimado (Gelman y Gottfried, 1996), y la generalización de conocimiento de propiedades no visibles en juego exploratorio (Baldwin, Markman, y Melartin, 1993). Estos estudios han mostrado la creciente capacidad de los niños para generar un conocimiento a través de la experimentación que es inherente a las situaciones de resolución de problemas.

Sin embargo, aunque el desempeño de los niños mejora, en relación con la actividad generada y con capacidades otorgadas por el desarrollo cognitivo, los argumentos utilizados por los niños sobre el conocimiento muestran una relación particular con las características de las situaciones que resuelven. Tal y como lo proponen Flavell, Mumme, Green y Flavell (1992) los niños crean una transformación sobre su propio conocimiento en tanto logra diferenciarlo del ajeno. 
Estos argumentos entonces, dan cuenta de la forma como los niños hallan la diferencia entre su propio pensamiento y el ajeno, y sus diferentes estados de conocimiento (Kuhn, Cheney y Weinstock, 2000a), analizando simultáneamente su validez (Pillow, Hill, Boyce y Stein, 2000). Esta capacidad para pensar sobre el conocimiento y sus formas de adquisición, es lo que Hofer y Pintrich (1997) llaman teorías epistemológicas, y adquiere relevancia, pues incluye la argumentación de los niños en torno a diferentes estados de conocimiento.

Estas teorías son lo que se ha llamado comprensión epistemológica, que es la capacidad para coordinar dimensiones subjetivas y objetivas de conocimiento (Kuhn et al., 2000a). Se dice que un conocimiento está en una dimensión objetiva en tanto el sujeto cognoscente considera que el conocimiento puntual sobre un tema especifico es una entidad localizada en el mundo real y por lo tanto conocible con certeza, independiente del observador que accede a dicho conocimiento.

Una dimensión subjetiva de conocimiento es aquella en la cual el peso de la validez del conocimiento pasa de un objeto cognoscible a un sujeto cognoscente, lo que genera una conciencia de la incertidumbre del conocimiento en tanto su origen se da en mentes humanas. Este paso tiene consecuencias positivas para la educación debido a que introduce la capacidad para comparar opiniones divergentes en torno a una misma problemática, dando paso a la posibilidad de un pensamiento critico sobre diferentes argumentos.

La coordinación de dimensiones reintegra el aspecto objetivo del conocimiento y reconoce la incertidumbre originada por la mente humana. Así, dos opiniones pueden ser legítimas y válidas pero una de las dos ha de serlo más, siendo la evidencia lo que respaldará uno u otro argumento. La coordinación entre dimensiones se alcanza cuando se reconoce que la realidad no es cognoscible directamente pero puede ser evaluada de acuerdo a criterios de argumentos y evidencia. Es allí donde la evaluación de evidencia se hace un proceso imprescindible.

Esta conceptualización acerca de la relación sujeto - objeto - conocimiento, está puesta en los procesos que subyacen en creación por parte del niño, de un mundo donde el origen determina la pertinencia de un conocimiento determinado. A medida que el desarrollo cognitivo tiene efecto, el conocimiento pasa a ser objeto de evaluación a través del proceso que le da forma y de la evidencia que lo sustenta. 
Es en este sentido donde el sujeto de conocimiento tiene un papel creador y activo al inyectarle sentido y validez a la realidad que percibe, en él mismo y en otros. Esta conceptualización sobre el mundo da origen a la idea que las conceptualizaciones epistemológicas siempre dependerán de los procesos metacognitivos que un sujeto de conocimiento posea en un determinado momento. Kuhn y Pearsall (2000b) han intentando demostrar que el nivel epistemológico de las personas, unidos con las demandas inherentes de cada situación experimental, ejerce una influencia sobre la manera como usamos nuestras destrezas intelectuales, e incluso influencian la adquisición de nuevo conocimiento.

Planteo entonces que la resolución de situaciones de tipo práctico y funcional estaría basada en procesos de autorregulación, pues las acciones parten de un conocimiento en su mayoría implícito pero susceptible al impacto que tengan sobre el material y volviéndose así explicito a medida que se interactúa con la situación, siendo esta una primera hipótesis de trabajo.

La comprensión epistemológica está en estrecha relación con la forma como las personas se pueden distanciar de su propio conocimiento y evaluar un determinado fenómeno desde la perspectiva de otro, la información de retorno que proveerán las situaciones debería modificar sus argumentos al permitirles evaluar las creencias de los personajes, siendo esta una segunda hipótesis de trabajo; queda para el futuro analizar la correlación existente entre autorregulación y comprensión epistemológica.

Para dar respuesta a estas hipótesis se planteó como objetivo general determinar las transformaciones en las concepciones implícitas de carácter físico en niños entre 5.0 a 5.5 años de edad cuando resuelven problemas isomórficos de índole inferencial e inductiva. De los diversos niveles que conlleva la situación, que integra acciones para resolverla y verbalizaciones para evaluar un estado de conocimiento, se desprenden dos objetivos específicos:

a) Analizar la capacidad de los niños para resolver situaciones problema y evaluar la evidencia que estas proveen, siendo sus estrategias un índice de autorregulación, y

b) Examinar los niveles de comprensión epistemológica de los argumentos utilizados para describir el estado de conocimiento de los personajes de la situación. 


\section{Método}

\section{Sujetos}

La población esta conformada por 120 niños y niñas, de tres instituciones de educación preescolar de carácter privado de estrato socioeconómico 6 de la ciudad de Santiago de Cali, siendo éste el estrato más alto en esta clasificación hecha por las Empresas Públicas de la ciudad. La muestra final estuvo conformada por 40 niños y niñas con una edad entre 5.0 y 5.5 años de edad seleccionados al azar a través de un programa de computador (SPSS), en esta selección sólo participaron los niños que recibieron autorización escrita de sus respectivos padres de familia.

\section{Situaciones y Material Experimental}

El material experimental esta basado en una situación de Chen y Klahr (1999). Consta de dos rampas movibles que originan cuatro diferentes grados de inclinación $\left(15^{\circ}, 30^{\circ}, 45^{\circ}\right.$, $60^{\circ}$ ), una de ellas tiene un tercio de su superficie cubierto por un plástico no translúcido, en la superficie oculta tiene ubicados reductores de velocidad que hacen que una mayor aceleración produzca sobresaltos en el recorrido retrasando así su llegada, solo quien genere una aceleración constante a partir de la inclinación de una de las rampas podrá darle solución a la situación.

Las situaciones están relacionadas con la comprobación empírica de las creencias proporcionadas a partir de dos personajes que semejan ser mecánicos de automóviles de F1, la acción sobre el material genera evidencia que da cuenta de la veracidad de las propuestas de índole mecánica de los personajes y es donde la autorregulación tiene un sentido, esta veracidad esta basada en la comprobación empírica que realiza el niño que resuelve la situación. Dichas creencias son una medida de control al restringir la formulación de hipótesis que se originaría si se buscase el fenómeno causal del funcionamiento de los vehículos.

Una situación principal, y tres situaciones isomorficas en las cuales se utilizaba el mismo material pero ambientación diferente, fueron presentadas bajo el supuesto que, al contener una estructura común, habrían de exigir a quien las resolviera, la creación y uso de 
inferencias de tipo inductivo y funcional. Así puede evaluarse la comprensión alcanzada para cada situación controlando huellas de aprendizaje originadas por la primera resolución.

El dominio específico en el cual puede inscribirse esta situación es el físico, entendido como un conjunto de representaciones que sostiene un conocimiento dado (Karmiloff-Smith, 1992), del cual existe una comprensión básica pero temprana de propiedades dinámicas y no requieren la inserción del sujeto en un medio escolar formal y/o avanzado. La relación empuje-velocidad que será abordada en la situación experimental es verificada en una situación de familiarización.

\section{Diseño y Plan Experimental}

El diseño incluyó un grupo experimental y uno control (denominados grupo 1 y 2 respectivamente), la variable independiente es el número de situaciones, cuatro al grupo experimental y dos al control, las cuales fueron presentadas en sendas sesiones con un intervalo de una hora, se controló además la edad de los participantes. Las consignas de la situación eran: "algo ha pasado, los carros tenían que haber llegado al mismo momento. El primer mecánico cree que es un problema de la pista y quiere cambiarla y un segundo mecánico cree que es problema del arranque, y quiere empujarlo mas duro, ¿será que es verdad?, tienes que ayudarlos a saber si eso es verdad o no".

Seguidamente el niño realizaba la actividad modificando a voluntad las características de las pistas, posteriormente se realizaba la pregunta control con el fin de determinar la comprensión de la tarea por parte del niño: “¿recuerdas lo que creía este mecánico? ¿Era verdad lo que decía o no lo era?". Los datos presentados reúnen solo los sujetos que respondieron afirmativamente y correctamente a la primera pregunta.

Se distribuyeron los sujetos en dos grupos: Al primer grupo se les presentan las cuatro situaciones y al segundo solo dos y es considerado un grupo control; las comparaciones entre los dos grupos generan la posibilidad de identificar y medir las variaciones debidas al aprendizaje.

\section{Categorías de análisis}


Las categorías de análisis de la actividad resolutoria están relacionadas con la capacidad de generar y trabajar con la información que surja de su interacción con el material experimental (Ordóñez, 2000) y establecen una jerarquía frente a la autorregulación alcanzada a lo largo de las sesiones:

Categoría A: Establecer relaciones entre elementos constitutivos. Este criterio hace referencia al tipo de relaciones de índole física que se establecen al accionar sobre elementos del material experimental, llamados constitutivos. Establecer dichas relaciones conlleva una identificación de las relaciones directamente proporcionales entre las variables físicas, del tipo: a menor ángulo-menor velocidad, mayor fuerza-mayor velocidad. Implica inicialmente una actividad exploratoria e inductiva sobre el material pero posteriormente es la anticipación la que limita necesariamente el tipo de acciones sobre las rampas al comprender que cierto tipo de acción genera resultados específicos.

Categoría B: Uso de información relevante. Esta categoría da cuenta de la capacidad de lograr en los carros el desempeño solicitado en la rampa con reductores de velocidad, mediante la manipulación de los elementos presentes en la situación. Implica una capacidad que alude a inferencias inductivas y funcionales pero antecede a la relación creenciaevidencia.

Categoría C: Evaluación de evidencia. Esta categoría se considera de índole superior y contiene las categorías anteriormente enumeradas. Si después de presenciar diferencias entre los comportamientos de ambos autos y lograr los resultados solicitados por la situación, evalúa la veracidad de las posibles respuestas al problema original (creencias de los personajes) seleccionando la correcta se considera un indicador suficiente de la posibilidad de evaluar sus acciones y el impacto de las mismas.

La clasificación sobre comprensión epistemológica esta basada en las propuestas de Hofer y Pintrich (1997) y surgen de las justificaciones y argumentos de los niños que estén relacionadas con las creencias de los personajes, generando tres niveles: a) En el nivel absoluto los sujetos reconocen, con base en el conocimiento que poseen, la posibilidad de generarse en la mente de otro una falsa creencia sobre un evento en particular, b) En el nivel 
múltiple la realidad no es cognoscible directamente y esta mediada por las creencias y conocimientos de las mentes humanas, haciendo el conocimiento que provenga de ellas incierto y c) En el nivel evaluativo se reconoce que la realidad no es cognoscible directamente pero puede ser evaluada de acuerdo a criterios de argumentos y evidencia.

\section{Resultados}

Los datos están en función de las dos hipótesis planteadas y solo se presentan los obtenidos en las sesiones 1 y 4 . Para la primera se presenta el promedio de acciones exitosas por categoría, siendo estas las que producían un resultado adecuado para la solución de la tarea y son consideradas un índice de autorregulación, en torno a la segunda hipótesis los datos se presentan a partir de los niveles de comprensión epistemológica. Luego de comprobar homocedasticidad, se aproximó a la variable normal y se realizó $\mathrm{X}^{2}$ Chi cuadrado utilizando la corrección de continuidad de Yates.

Tabla 1. Promedio de acciones exitosas por categorías y grupos

\begin{tabular}{|l|c|c|c|c|c|c|}
\hline & \multicolumn{6}{|c|}{ Tipo de Acciones Sobre el Material } \\
\hline & \multicolumn{2}{|c|}{ A } & \multicolumn{2}{c|}{ B } & \multicolumn{2}{c|}{ C } \\
\hline Sesion & Grupo 1 & Grupo 2 & Grupo 1 & Grupo 2 & Grupo 1 & Grupo 2 \\
\hline 1 & 11.5 & 13.7 & 10.4 & 11.1 & 2.4 & 1.8 \\
\hline 4 & 20.8 & 15.4 & 24.6 & 14.7 & 8.1 & 3.7 \\
\hline
\end{tabular}

La tabla 1 presenta el promedio de acciones exitosas en la resolución de la tarea por sesiones. Para el grupo control y en función de la variable independiente solo hubo dos sesiones. El promedio aumentó significativamente entre sesiones para el grupo experimental $\left(X^{2}=4,01\right.$, g.1.: $1, \mathrm{p}<0.05$ para la categoría $\mathrm{A}, X^{2}=4,27$, g.1.: $1, \mathrm{p}<0.05$ para la categoría $\mathrm{B}, \mathrm{y}$ $X^{2}=3,98$, g.l.: $1, \mathrm{p}=0.05$ para la categoría C); para el grupo control existe una tendencia al alza aunque no llega a ser significativa en ninguna categoría.

No se encontraron diferencias estadísticamente significativas con $X^{2}$ entre ambos grupos en la primera sesión $\left(X^{2}=2.90\right.$ y $\left.X^{2}=2.78\right)$. Para determinar la significancia de las diferencias entre ambos grupos en la sesión final se utilizó la prueba Mann-Whitney para dos grupos con datos independientes encontrando que son significativamente mayores que las esperadas debido al azar con un riesgo de 5\% en las tres categorías, particularmente en la 
categoría $\mathrm{C}(U=[1550,5 ; 130.5], \mathrm{p}<.05)$ donde se es más clara la posibilidad de los niños para evaluar la evidencia disponible.

Tabla 2. Niveles epistemológicos a través de las sesiones (solamente grupo 1)

\begin{tabular}{|c|c|c|c|}
\hline \multicolumn{4}{|c|}{ Porcentajes de participantes que mostraron un nivel predominantemente absolutista } \\
\hline Sesión 1 & Sesión 2 & Sesión 3 & Sesión 4 \\
\hline $90 \%$ & $85 \%$ & $90 \%$ & $80 \%$ \\
\hline Porcentajes de participantes que mostraron un nivel predominantemente evaluativo \\
\hline Sesión 1 & Sesión 2 & Sesión 3 & Sesión 4 \\
\hline $10 \%$ & $15 \%$ & $5 \%$ & $20 \%$ \\
\hline
\end{tabular}

La tabla 2 presenta el porcentaje de sujetos ubicados en los respectivos niveles de comprensión epistemológica; no se encontraron expresiones que fueran indicio de la presencia de un nivel múltiple. Los resultados muestran que las justificaciones de carácter absoluto tienen una estabilidad en los tipos de argumentos para describir el conocimiento de los personajes de la situación 1 a lo largo de las diferentes sesiones, sin embargo las justificaciones de carácter evaluativo si sufrieron fluctuaciones.

\section{Discusión}

En función de los resultados obtenidos y las hipótesis planteadas se pueden discutir dos temáticas: a) la relación entre las concepciones implícitas, y la resolución de problemas isomórficos de carácter inferencial y funcional, y b) los argumentos de los niños en torno al conocimiento de los personajes y su veracidad, siendo estos un indicador de sus niveles de comprensión epistemológica.

Los datos apoyan la primera hipótesis, efectivamente en la resolución reiterada de situaciones de tipo práctico y funcional, las acciones emprendidas por los niños en la búsqueda de la solución se organizan en series más complejas y con una efectividad mayor. Las relaciones entre las verbalizaciones de los personajes y la efectividad de sus propias 
secuencias de acción, se hacen explicitas para los niños, añadiendo así características metacognitivas.

De tal forma los procesos de autorregulación identificados estuvieron basados en un cambio en las verbalizaciones de los niños pasando de mencionar los acciones emprendidas sobre el material, a controlar las acciones, a través del establecimiento de relaciones entre las acciones a emprender y las realizadas con anterioridad, y su respectiva efectividad. Tal y como lo propone Lacasa et al. (1995) los niños se sitúan en diferentes niveles de abstracción para controlar su propia actividad; en este sentido la incorporación de la planificación se convierte en un recurso de carácter metacognitivo.

Recientemente, Kuhn (2001) ha abordado el estudio de procesos metacognitivos proponiendo una distinción entre el conocimiento meta-cognitivo (metacognitive knowing) y conocimiento meta-estratégico (metastrategic knowing) siendo su característica distintiva en el primero el ser un conocimiento declarativo y del segundo estar basado en el conocimiento de la tarea y la efectividad de las estrategias disponibles para alcanzar la solución.

Desde esta clasificación, se encuentran interesantes vínculos en la autorregulación desarrollada a lo largo de las sesiones, pues en estas se origina un conocimiento declarativo que paulatinamente le da sentido a la acción realizada. Desde una perspectiva basada en procesos cognitivos, al aumentar el número de sesiones, la acción que era índice de la inferencia inductiva resultó indiscutible, en la actividad aparecieron relaciones en el material propuesto que para los niños no eran evidentes en un comienzo, superando así la información dada y extrayendo un conocimiento nuevo, siendo esto el atributo típico de la inferencia.

¿Pero autoriza solo una resolución exitosa el hablar de inferencia?. La relación con la autorregulación que se discute en este artículo lleva a pensar que solamente cuando el sujeto puede utilizar la información dada a partir de los elementos de la situación, y descubre algo nuevo de manera explicita es que se puede hablar de una resolución a través de inferencias inductivas, siendo en este caso la identificación de la relación física propuesta en la tarea su indicador.

Thornton (1998) ha propuesto que los niños desde muy temprano presentan comportamientos a los cuales es posible atribuírseles un componente inferencial, permitiéndoles depender de su propio razonamiento y no de las características de la tarea. De 
igual forma los niños pasaron a guiarse por la información que ellos elaboraron y no por los elementos perceptuales de la tarea.

La actividad de los niños dejó entrever la fortaleza y características de las concepciones físicas elaboradas por los participantes. En la sesión de inicio y de manera recurrente (61.5\% de las acciones promediando ambos grupos) se consideraba que el empuje y su correspondiente resultado -velocidad- era requisito único y suficiente para solucionar el problema, siendo esta la primera concepción implícita encontrada en el plano físico. El hecho de pasar rápidamente por los reductores causaba sobresaltos que disminuían la velocidad, solo quien infería que una aceleración paulatina era capaz de resolver exitosamente la tarea.

Schauble (1996) ha analizado el comportamiento y creencias de los preescolares frente al dominio físico y en particular en hidrodinámica, encontrando que los preescolares son renuentes a abandonar estrategias o concepciones inválidas, a pesar de que en la experiencia han comprobado su inutilidad; además sus concepciones se transforman durante las sesiones en mayor proporción que los adultos.

En esta investigación se halló por el contrario, que paulatinamente los niños infirieron que la aceleración era relevante para superar los reductores de velocidad de la rampa (del $22.5 \%$ de los intentos en la primera sesión al $42.8 \%$ en la segunda). Probablemente la mecánica y el movimiento rectilíneo son más transparentes que la hidrodinámica al ser más perceptible en un mundo práctico. Estos resultados resultan ser muestra de la capacidad para evaluar evidencia por parte de los niños.

Los resultados entonces contradicen abiertamente propuestas como las de Kuhn, Amsel y O'Loughlin (1988) cuando afirmaron que los niños pequeños usan la evidencia a favor de sus propias teorías o usan la evidencia para construir nuevas teorías, pero fallan en comprender cómo esas nuevas teorías difieren de las primeras. Frente a esta situación los niños resultaron ser efectivos en transformar sus concepciones a través de la evaluación de evidencia.

Sin embargo, es interesante analizar que estatuto le podemos otorgar a las creencias de carácter físico que presentaron los sujetos, ¿pueden ser consideradas teorías tal como lo afirman diversos autores? (Vosniadou, 2002; Pozo et al., 1992). En primer lugar se debe tener 
en cuenta que al hablar de teoría no se pretende que esta la sea en un sentido explicito. Gopnik y Wellman (2002) han propuesto que a los cinco años se poseen constructos generales que resultan ser explicativos, predictivos y que están basados en creencias y no en deseos, lo cual es característico de edades menores.

Dos resultados permiten dar un cierto estatus teórico a las ideas de los participantes, una única variable en el movimiento rectilíneo resulto ser la velocidad, que era suficiente para realizar proyecciones sobre el movimiento de los vehículos y su alcance en la rampa. Sin embargo, fue sensible al cambio, los argumentos dejaron ver a través de su contenido que son creencias en torno al conocimiento, lo que explica la actividad.

En contraste a los grandes cambios que se observaron en torno a la actividad inferencial y autorreguladora, en los argumentos utilizados y sus niveles de comprensión epistemológica no se encontró mayor cambio, por lo tanto no se encontró un apoyo empírico a la segunda hipótesis ubicándose en su mayoría en un nivel absoluto. Kuhn y Pearsall (2000b) han propuesto que los inicios de las capacidades metacognitivas relacionadas con la coordinación de dimensiones de conocimiento, han de buscarse en la Teoría de la Mente pues es necesario comprender que alguien puede tener diversas creencias sobre un evento y que estas pueden diferir de las propias.

Si bien la edad de los participantes hace pensar que una teoría mentalista ya este presente, los participantes tuvieron dificultad en relacionar la información que la situación proporcionaba con las creencias originales del personaje, resultados que refuerzan investigaciones anteriores. En un estudio de Kuhn et al. (2000a) en el cual se indagó la importancia del tipo de dominio de conocimientos y su relación con el desarrollo cognitivo en juicios epistemológicos, se encontró que el $75 \%$ de los niños de $5^{\text {to }}$ grado se ubicaron en un nivel absoluto.

La solución reiterada de problemas isomórficos no resulto ser efectiva para transformar sus argumentos y la relación que tenían estos con la información hallada en la solución de la tarea. En este caso y como hipótesis explicativa, las restricciones de carácter cognitivo se impusieron y evitaron que se produjera un distanciamiento entre las creencias de 
los personajes y de los participantes. Es necesario en un futuro analizar de manera correlacional la actividad resolutoria y los argumentos de los niños.

Aún cuando se ha sostenido (Chen y Klahr, 1999) que la instrucción apropiada es un requisito indispensable para que niños de escuela elemental aprendan y transfirieran estrategias básicas cuando evalúan diseños experimentales, y se reconoce que no se explora la relación entre metodología y comprensión a largo plazo, este estudio muestra que una situación donde se realice un análisis inductivo a través de la resolución de situaciones isomorficas, mejora significativamente la física intuitiva de preescolares y enriquece aun más la maravillosa experiencia de estar en preescolar.

\section{Referencias}

Baldwin, D., Markman, E., y Melartin, R. (1993). Infants' ability to draw inferences about nonobvious object properties: Evidence from exploratory play. Child Development, $64,711-728$.

Chen, Z., y Klahr, D. (1999). All other things being equal: Acquisition and transfer of the control of variable strategy. Child Development, 70, 1098-1120.

Flavell, J., Mumme, D., Green, F., y Flavell, E. (1992). Young children's understanding of different types of beliefs. Child Development, 63, 960-977.

Florian, J. (1994). Stripes do not a zebra make, or do they? Conceptual and perceptual information in inductive inference. Developmental Psychology, 30, 88-101.

Gelman, S., y Gottfried, G. (1996). Children's explanation of animate and inanimate motion. Child Development, 67, 1971-1987.

Gopnik, A., y Wellman, H. (2002). La teoría de la teoría. En A. Hirschfeld, y S. Gelman (Comps), Cartografía de la Mente (p. 221- 243). Barcelona: Gedisa, S.A. (Orig. 1994).

Hofer, B., y Pintrich, P. (1997). The development of epistemological theories: Beliefs about knowledge and knowing and their relation to learning. Review of Educational Research, 67, 88-140.

Johnson-Laird, P. (1994). A model theory of induction. International Studies in the Philosophy of Science. 8, 5-29.

Karmiloff-Smith, A. (1992). Más allá de la modularidad. Madrid: Alianza Editorial. 
Kuhn, D., Amsel, E., y O'Loughlin, M. (1988). The development of scientific thinking skills. Orlando, FL: Academic Press, Inc.

Kuhn, D. (2001). How do people know?. Psychological Science, 12, 1-8.

Kuhn, D. (2002). Metacognitive development. Current Directions in Psychological Science, 9, 178-181.

Kuhn, D., y Pearsall, S. (2000b). Developmental origins of scientific thinking. Journal of Cognition and Development, 1, 113-129.

Kuhn, D., Cheney, R., y Weinstock, M. (2000a). The development of epistemological understanding. Cognitive Development, 15, 309-328.

Lacasa, P., Martín, B., y Herranz, P. (1995). Autorregulación y relaciones entre iguales en tareas de construcción: Un análisis de las situaciones de interacción. Infancia y Aprendizaje, 72, 71-94.

Niaz, M. (1995). Enhancing thinking skills: Domain specific / domain general strategies. Instructional Science, 22, 413 - 422.

Oliva, J. (1999). Concepciones de los alumnos en física y diferencias individuales. Infancia y Aprendizaje, 88, 3-24.

Ordóñez, O. (2000). Inferencia y comprensión en niños entre 3 a 5 años. Un estudio piloto. Cali: Universidad del Valle.

Pauen, S. (1996). Children's reasoning about the interaction of forces. Child Development, 67, 2729-2742.

Penner, D., y Klahr, D. (1996). The interaction of domain-specific knowledge and domaingeneral strategies: A study whit sinking objects. Child Development, 67, 2709-2727.

Pillow, B., Hill, V., Boyce, A., y Stein, C. (2000). Understanding inference as a source of knowledge: Children's ability to evaluate the certainty of deduction, perception, and guessing. Developmental Psychology, 36, 169- 179.

Pozo, J. (1993). Psicología y didáctica de las ciencias de la naturaleza: ¿Concepciones alternativas?. Infancia y Aprendizaje, 62-63, 187-204.

Pozo, J., Sanz, A., y Limón, M. (1992). Las ideas de los alumnos sobre la ciencia como teorías implícitas. Infancia y Aprendizaje, 57, 3-22.

Schauble, L. (1996). The development of scientific reasoning in knowledge-rich contexts. Developmental Psychology, 32, 102-119.

Thornton, S. (1998). La resolución infantil de problemas. Madrid: Morata. 
Vosniadou, S. (2002). Propiedades universales y culturo-específicas de los modelos mentales de los niños acerca de la tierra. En A. Hirschfeld, y S. Gelman (Comps), Cartografía de la Mente (p. 221- 243). Barcelona: Gedisa, S.A. (Orig. 1994). 